\title{
Zenglv Fumai Granule protects cardiomyocytes against hypoxia/reoxygenation-induced apoptosis via inhibiting TRIM28 expression
}

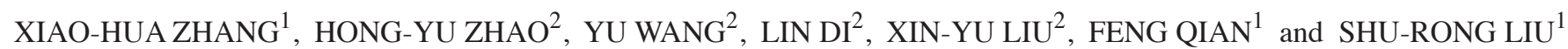 \\ ${ }^{1}$ Department of Cardiovascular Diseases, The First Clinical Hospital of Jilin Academy of \\ Chinese Medicine Sciences; ${ }^{2}$ Drug Pharmacology and Toxicology Evaluation Center, \\ Jilin Academy of Chinese Medicine Sciences, Changchun, Jilin 130021, P.R. China
}

Received April 14, 2020; Accepted November 23, 2020

DOI: $10.3892 / \mathrm{mmr} .2020 .11810$

\begin{abstract}
Myocardial ischemia/reperfusion (MIR) injury, which occurs following acute myocardial infarction, can cause secondary damage to the heart. Tripartite interaction motif (TRIM) proteins, a class of E3 ubiquitin ligases, have been recognized as critical regulators in MIR injury. Zenglv Fumai Granule (ZFG) is a clinical prescription for the treatment of sick sinus syndrome, a disease that is associated with MIR injury. The present study aimed to investigate the effect of ZFG on MIR injury and to determine whether ZFG exerts its effects via regulation of TRIM proteins. In order to establish an in vitro MIR model, human cardiomyocyte cell line AC16 was cultured under hypoxia for $5 \mathrm{~h}$ and then under normal conditions for $1 \mathrm{~h}$. Following hypoxia/reoxygenation (H/R) treatment, these cells were cultured with different ZFG concentrations. ZFG notably inhibited H/R-induced cardiomyocyte apoptosis. The expression levels of four TRIM proteins, TRIM7, TRIM14, TRIM22 and TRIM28, were also detected. These four proteins were significantly upregulated in $\mathrm{H} / \mathrm{R}$-injured cardiomyocytes, whereas their expression was inhibited following ZFG treatment. Moreover, TRIM28 knockdown inhibited H/R-induced cardiomyocyte apoptosis, whereas TRIM28 overexpression promoted apoptosis and generation of reactive oxygen species (ROS) in cardiomyocytes. However, the effects of TRIM28 overexpression were
\end{abstract}

Correspondence to: Dr Shu-Rong Liu, Department of Cardiovascular Diseases, The First Clinical Hospital of Jilin Academy of Chinese Medicine Sciences, 1745 Gongnong Road, Chaoyang, Changchun, Jilin 130021, P.R. China

E-mail: z15568853807@163.com

Abbreviations: MIR, myocardial ischemia/reperfusion; TRIM, tripartite interaction motif; H/R, hypoxia/reoxygenation; NAC, $\mathrm{N}$-acetyl-L-cysteine; ROS, reactive oxygen species

Key words: apoptosis, TRIM28, glutathione peroxidase 1, Zenglv Fumai Granule limited by the action of ROS inhibitor N-acetyl-L-cysteine. In addition, the mRNA and protein levels of antioxidant enzyme glutathione peroxidase (GPX)1 were significantly downregulated in H/R-injured cardiomyocytes. TRIM28 knockdown restored GPX1 protein levels but had no effect on mRNA expression levels. Co-immunoprecipitation and ubiquitination assays demonstrated that TRIM28 negatively regulated GPX1 via ubiquitination. In sum, the present study revealed that $\mathrm{ZFG}$ attenuated H/R-induced cardiomyocyte apoptosis by regulating the TRIM28/GPX1/ROS pathway. ZFG and TRIM28 offer potential therapeutic options for the treatment of MIR injury.

\section{Introduction}

Coronary artery disease, which caused $>9$ million deaths in 2016, is a leading cause of death worldwide (1). Early reperfusion therapy is the most effective strategy in the treatment of myocardial infarction, a common type of coronary artery disease (2). However, the process of reperfusion can induce myocardial ischemia/reperfusion (MIR) injury, a complex pathophysiological process that involves oxidative stress, $\mathrm{Ca}^{2+}$ overload and inflammatory response $(3,4)$, eventually leading to programmed cell death of cardiomyocytes (5-7). In order to improve the beneficial effects of reperfusion therapy, it is necessary to develop powerful and safe drugs for the treatment for MIR injury.

Chinese herbal medicine has been used in the treatment of MIR injury for hundreds of years (8). Numerous herbal formulas have been determined to protect cardiomyocytes from MIR injury (9-11). Zenglv Fumai Granule (ZFG), a clinical discovery by Professor Yu Zuo-Ying, has been used in the treatment of sick sinus syndrome for $>30$ years (12). Sick sinus syndrome is a disorder characterized by abnormal heart rhythms due to the malfunction of the sinus node (13). Our previous study revealed that $\mathrm{ZFG}$ decreases clinical symptoms and improves heart rate in patients with sick sinus syndrome (12). A previous study demonstrated that sick sinus syndrome is associated with MIR injury (14). Therefore, it was hypothesized that ZFG may also serve a protective role against MIR injury. 
Tripartite interaction motif (TRIM) proteins are a class of E3 ubiquitin ligases that contain a ring-finger domain, one or two B-boxes and a coiled-coil region at the amino-terminal region (15). They function via the regulation of protein degradation by ubiquitination, and are thereby involved in numerous biological processes, such as proliferation and apoptosis (16). Previous studies have revealed that TRIM proteins are key regulators in MIR injury $(17,18)$. For example, TRIM6 aggravates MIR injury by inducing cardiomyocyte apoptosis (17) and TRIM59 alleviates MIR injury via the inhibition of myocardial apoptosis and inflammatory response (18). A recent study demonstrated aberrant expression levels of multiple TRIM proteins in the heart during myocardial infarction, including TRIM7, TRIM14, TRIM22 and TRIM28 (19); however, it is unclear whether they serve a role in MIR injury.

The present study aimed to investigate the effect of ZFG on MIR-induced myocardial apoptosis, and to determine whether it functions via the regulation of TRIM proteins. An in vitro MIR injury model was established by treating human cardiomyocyte cell line AC16 with hypoxia/reoxygenation (H/R). Using this model, the hypothesis that ZFG had a protective role in MIR injury by regulating the TRIM28/GPX1 axis was tested.

\section{Materials and methods}

Extraction of ZFG. ZFG consists of eight types of herbal medicines, including Panax ginseng (radix), Astragalus propinquus (radix), Cinnamomum cassia (ramulus), Epimedium brevicornum (aerial part), Polygonatum sibiricum (rhizoma), Salvia miltiorrhiza (radix), Ligusticum chuanxiong (rhizome) and Ophiopogon japonicas (radix) (12). These herbs were mixed in the following ratio: $4: 6: 3: 4: 4: 4: 3: 4$. Herbs were decocted twice ( $2 \mathrm{~h}$ each time) with 10 times the amount of water, as previously described (20). The extracts were made into lyophilized powder for subsequent experiments.

Cell culture. Human cardiomyocyte cell line AC16 was purchased from The Cell Bank of Type Culture Collection of The Chinese Academy of Sciences. AC16 cells were cultured in DMEM (HyClone; Cytiva) with $10 \%$ fetal bovine serum (Gibco; Thermo Fisher Scientific, Inc.) and $1 \%$ penicillinstreptomycin solution (Beijing Solarbio Science \& Technology Co., Ltd.) in a humidified incubator at $5 \% \mathrm{CO}_{2}$ at $37^{\circ} \mathrm{C}$.

$H / R$ treatment. $\mathrm{H} / \mathrm{R}$ treatment was performed as previously described by Benoist et al (21). In brief, AC16 cells were cultured in glucose/serum-free DMEM (Gibco; Thermo Fisher Scientific, Inc.) under hypoxia $\left(95 \% \mathrm{~N}_{2}\right.$ and $\left.5 \% \mathrm{CO}_{2}\right)$ for $5 \mathrm{~h}$ at $37^{\circ} \mathrm{C}$. Then, cells were transferred to normal medium and cultured under reoxygenation $\left(5 \% \mathrm{CO}_{2}\right.$ and $\left.95 \% \mathrm{O}_{2}\right)$ for $1 \mathrm{~h}$ at $37^{\circ} \mathrm{C}$. Cells that were cultured under normal conditions only were used as the control. Following H/R treatment, various concentrations of ZFG $(0.0,0.1,0.2$ and $0.4 \mathrm{mg} / \mathrm{ml})$ were added and cells were incubated for $24 \mathrm{~h}$ at $37^{\circ} \mathrm{C}$.

Lentivirus construction and transduction. Lentivirus overexpression (oe)TRIM28 and small interfering (si)TRIM28 were constructed in order to overexpress and knock down TRIM28, respectively. For constructing lentivirus oeTRIM28,
DNA fragments of TRIM28 were cloned into 1,000 ng vector plasmid pLVX-Puro (Clontech Laboratories, Inc.). Then, the second-generation packaging plasmids psPAX2 (100 ng) and pMD2G (900 ng; both Addgene, Inc.) were used to co-transfect the vector plasmid into 293 T cells using Lipofectamine ${ }^{\circledR} 2000$ (Invitrogen; Thermo Fisher Scientific, Inc.) at $37^{\circ} \mathrm{C}$. After $6 \mathrm{~h}$, the DMEM was replaced with complete medium. High-titer recombinant lentiviruses were obtained at 48 and $72 \mathrm{~h}$ after transfection. In order to construct lentivirus siTRIM28, the designed TRIM28 siRNAs (Generay Biotech Co., Ltd.) were cloned into the vector plasmid PLKO.1 (Addgene, Inc.), which was then transfected into $293 \mathrm{~T}$ cells as aforementioned. The sequences of TRIM28 siRNAs were as follows: siTRIM28-1, 5'-GCA ACAGTGCT TCTCCAAA-3'; siTRIM28-2, 5'-GGAGATGATCCCTACTCAA-3'; and siTRIM28-3, 5'-GGACTACAACCTTATTGTT-3'.

For lentivirus transduction, AC16 cells were inoculated into 6-well plates at a density of $2 \times 10^{5}$ cells/well. At $60-70 \%$ confluence, the cells were transduced with lentivirus at a multiplicity of infection of 10 at $37^{\circ} \mathrm{C}$ for $6 \mathrm{~h}$, followed by culture with fresh medium for a further $24 \mathrm{~h}$ at $37^{\circ} \mathrm{C}$. Cells transduced with lentivirus-containing vector plasmid or siNC sequence (5'-TCGCTGCATCAGATGAGAC-3') were used as negative controls. For subsequent experiments, cells were also cultured with $1 \mathrm{mmol} / \mathrm{l}$ ROS inhibitor N-Acetyl-L-cysteine (NAC) for $24 \mathrm{~h}$.

Measurement of apoptotic cells. AC16 cells were digested using $0.25 \%$ trypsin-EDTA solution (Beijing Solarbio Science \& Technology Co., Ltd.) and suspended in PBS for counting. Then, $1 \times 10^{5}$ cells were centrifuged at $500 \times \mathrm{g}$ for $5 \mathrm{~min}$ at $4^{\circ} \mathrm{C}$. The supernatant was discarded and cells were resuspended with $195 \mu 1$ Annexin V-FITC (Beyotime Institute of Biotechnology). Then, cells were incubated with $5 \mu \mathrm{l}$ Annexin V-FITC for $15 \mathrm{~min}$ in the dark at $4^{\circ} \mathrm{C}$, followed by incubation with $5 \mu \mathrm{l}$ propidium iodide for $5 \mathrm{~min}$ at $4^{\circ} \mathrm{C}$. Apoptotic cells were detected using BD Accuri ${ }^{\mathrm{TM}}$ C6 flow cytometer (BD Biosciences), and the data were analyzed by FlowJo software (v10.2.8; FlowJo LLC).

Reverse transcription-quantitative (RT-qPCR). Total RNA in AC16 cells was extracted using TRIzol ${ }^{\circledR}$ reagent (Invitrogen; Thermo Fisher Scientific, Inc.) and the residual DNA was digested using DNase I enzymes. The cDNA was synthesized using RevertAid First Strand cDNA Synthesis Kit (cat. no. K1622; Fermentas; Thermo Fisher Scientific, Inc.), according to the manufacturer's instructions. RT-qPCR was performed with SYBR-Green Mix (Thermo Fisher Scientific, Inc.) in an ABI 7300 Real-Time PCR System (Applied Biosystems; Thermo Fisher Scientific, Inc.). The thermocycling conditions were as follows: Initial denaturation at $95^{\circ} \mathrm{C}$ for $5 \mathrm{~min}$, followed by 45 cycles of $95^{\circ} \mathrm{C}$ for $15 \mathrm{sec}$ and $60^{\circ} \mathrm{C}$ for $45 \mathrm{sec}$. GAPDH was selected as the internal control. The relative mRNA levels of target genes (TRIM7, TRIM14, TRIM22, TRIM28 and GPX1) were calculated using the $2^{-\Delta \Delta C q}$ method (22). The primers are listed in Table I.

Western blotting. Total protein in AC16 cells was extracted using super RIPA lysis buffer (cat. no. R0010; Beijing Solarbio Science \& Technology Co., Ltd.), and its concentra- 
Table I. Primers used for reverse transcription-quantitative PCR.

\begin{tabular}{ll}
\hline Gene & \multicolumn{1}{c}{ Primer sequences $\left(5^{\prime} \rightarrow 3^{\prime}\right)$} \\
\hline GAPDH & F: AATCCCATCACCATCTTC \\
R: AGGCTGTTGTCATACTTC \\
FRIM7 \\
F: CTGAGGGCTTTCCTGGTG \\
TRIM14 & F: GAGCTTGTCGAGGGATGCG \\
& R: CTGGGTTATGTTGTCAATGTGC \\
TRIM22 & F: CAAACATTCCGCATAAAC \\
TRIM28 & R: ATCCAGCACATTCACCTCAC \\
& F: CCCGTCTTCAAGGTCTTCC \\
RPX1 & R: GAGCCATAAGCACAGGTTTG \\
& F: TCGGTGTATGCCTTCTCGG \\
& R: CTTGGCGTTCTCCTGATGC \\
\hline
\end{tabular}

F, forward; R, reverse; TRIM7, tripartite interaction motif 7; TRIM14, tripartite interaction motif 14; TRIM22, tripartite interaction motif 22; TRIM28, tripartite interaction motif 28; GPX1, glutathione peroxidase 1 .

tion was determined with a BCA protein assay kit (Thermo Fisher Scientific, Inc.). Then, $25 \mu \mathrm{g}$ protein was separated via SDS-PAGE on a $10 \%$ gel, and then transferred onto a PVDF membrane (EMD Millipore). After being blocked with $5 \%$ skimmed milk for $1 \mathrm{~h}$ at room temperature, the membrane was incubated with primary antibodies overnight at $4^{\circ} \mathrm{C}$, followed by incubation with HRP-conjugated secondary antibody (1:1,000; cat. no. A0208; Beyotime Institute of Biotechnology) for $1 \mathrm{~h}$ at $37^{\circ} \mathrm{C}$. The primary antibodies included antibodies against TRIM28 (1:1,000; cat. no. ab10484; Abcam), GPX1 (1:2,000; cat. no. ab108427; Abcam), cleaved caspase-3 (1:5,000; cat. no. ab214430; Abcam) and GAPDH (1:5,000; cat. no. 60004-1-1G; ProteinTech Group, Inc.). Finally, the PVDF membrane was washed with enhanced chemiluminescent detection reagent (EMD Millipore) and visualized using a Tanon-5200 system (Tanon Science and Technology Co., Ltd.). Densitometric analysis was performed using ImageJ software (v1.44; National Institutes of Health).

Detection of ROS levels. AC16 cells were incubated with $10 \mu \mathrm{M}$ 2'7'-dichlorofluorescin diacetate probe (Beyotime Institute of Biotechnology) for $20 \mathrm{~min}$ at $37^{\circ} \mathrm{C}$ in the dark. The fluorescence of AC16 cells was detected using a BD Accuri ${ }^{\text {TM }}$ C6 flow cytometer. Wavelengths of 480 and $525 \mathrm{~nm}$ were selected as the excitation and emission wavelengths, respectively.

Co-immunoprecipitation and ubiquitination assays. Total protein was extracted and its concentration was determined using BCA protein assay kit. Then, $2 \mathrm{mg}$ protein was incubated with $1 \mu \mathrm{g}$ antibodies against TRIM28, GPX1 or IgG (cat. no. 10283-1-AP; ProteinTech Group, Inc.) overnight at $4^{\circ} \mathrm{C}$. Thereafter, these samples were incubated with $30 \mu \mathrm{l}$ Protein A/G Plus-Agarose (Santa Cruz Biotechnology, Inc.) for $2 \mathrm{~h}$ at $4^{\circ} \mathrm{C}$. The supernatant was discarded following centrifugation at $4,500 \mathrm{xg}$ for $5 \mathrm{~min}$ at $4^{\circ} \mathrm{C}$. Then, the $\mathrm{A} / \mathrm{G}$ Plus-Agarose beads were washed four times with $1 \mathrm{ml}$ RIPA lysis buffer, and subsequently boiled with $2 \mathrm{X}$ loading buffer for $5 \mathrm{~min}$. Following centrifugation at $500 \mathrm{x} \mathrm{g}$ for $1 \mathrm{~min}$ at $4^{\circ} \mathrm{C}$, the supernatant was collected and western blotting was performed. Antibodies against TRIM28 or GPX1 were used as the primary antibodies.

For the ubiquitination detection of GPX1 protein, antiGPX1 antibody was used to pull down immunocomplex. Subsequent western blotting was performed according to the same method outlined above, but using anti-ubiquitin antibody (1:2,000; cat. no. ab7780; Abcam) as the primary antibody.

Statistical analysis. All experiments were repeated three times, and the experimental data are presented as the mean \pm SD. Statistical analysis was performed using Prism software (version 8.0.2; GraphPad Software Inc.). Statistical differences were evaluated by one-way ANOVA and post hoc Tukey's test. $\mathrm{P}<0.05$ was considered to indicate a statistically significant difference.

\section{Results}

ZFG inhibits H/R-induced apoptosis in AC16 cells. In order to investigate the effect of ZFG on myocardial apoptosis during $\mathrm{H} / \mathrm{R}$ injury, H/R-injured AC16 cells were treated with various ZFG dosages. The results revealed that $\mathrm{H} / \mathrm{R}$ treatment significantly increased apoptosis in AC16 cells, while subsequent ZFG administration significantly inhibited H/R-induced apoptosis in a dose-dependent manner (Fig. 1). In addition, $0.4 \mathrm{mg} /$ $\mathrm{ml}$ ZFG treatment-alone had no significant effect on apoptosis in AC16 cells (Fig. 1).

ZFG inhibits H/R-induced TRIM28 expression in AC16 cells. In order to determine whether TRIM proteins contributed to the protective effect exerted by ZFG, the expression levels of TRIM7, TRIM14, TRIM22 and TRIM28 in H/R-injured AC16 cells were assessed. H/R treatment significantly increased the mRNA expression levels of TRIM7, TRIM14, TRIM22 and TRIM28; these effects were eliminated by subsequent administration of ZFG (Fig. 2). Among the TRIM proteins, TRIM28 induced the greatest change in expression levels; therefore, it was selected for subsequent experiments. RT-qPCR and western blotting assays showed that TRIM28 mRNA and protein expression levels in H/R-injured AC16 cells significantly increased in a time-dependent manner (Fig. 3A and B), and subsequent ZFG treatment attenuated $\mathrm{H} / \mathrm{R}$-induced upregulation of TRIM28 expression levels in a dose-dependent manner (Fig. 3C and D).

Knockdown of TRIM28 inhibits H/R-induced apoptosis in AC16 cells. Lentivirus siTRIM28 was constructed to knock down TRIM28 expression in AC16 cells. All three siTRIM28 lentiviruses significantly decreased the mRNA and protein levels of TRIM28 (Fig. S1A and B). The lentiviruses siTRIM28-1 and siTRIM28-2 were then transduced into H/R-injured AC16 cells. Flow cytometric analysis revealed that TRIM28 knockdown attenuated H/R-induced upregulation of apoptotic rate in AC16 cells (Fig. 4A and B). Western blotting revealed that TRIM28 knockdown inhibited 


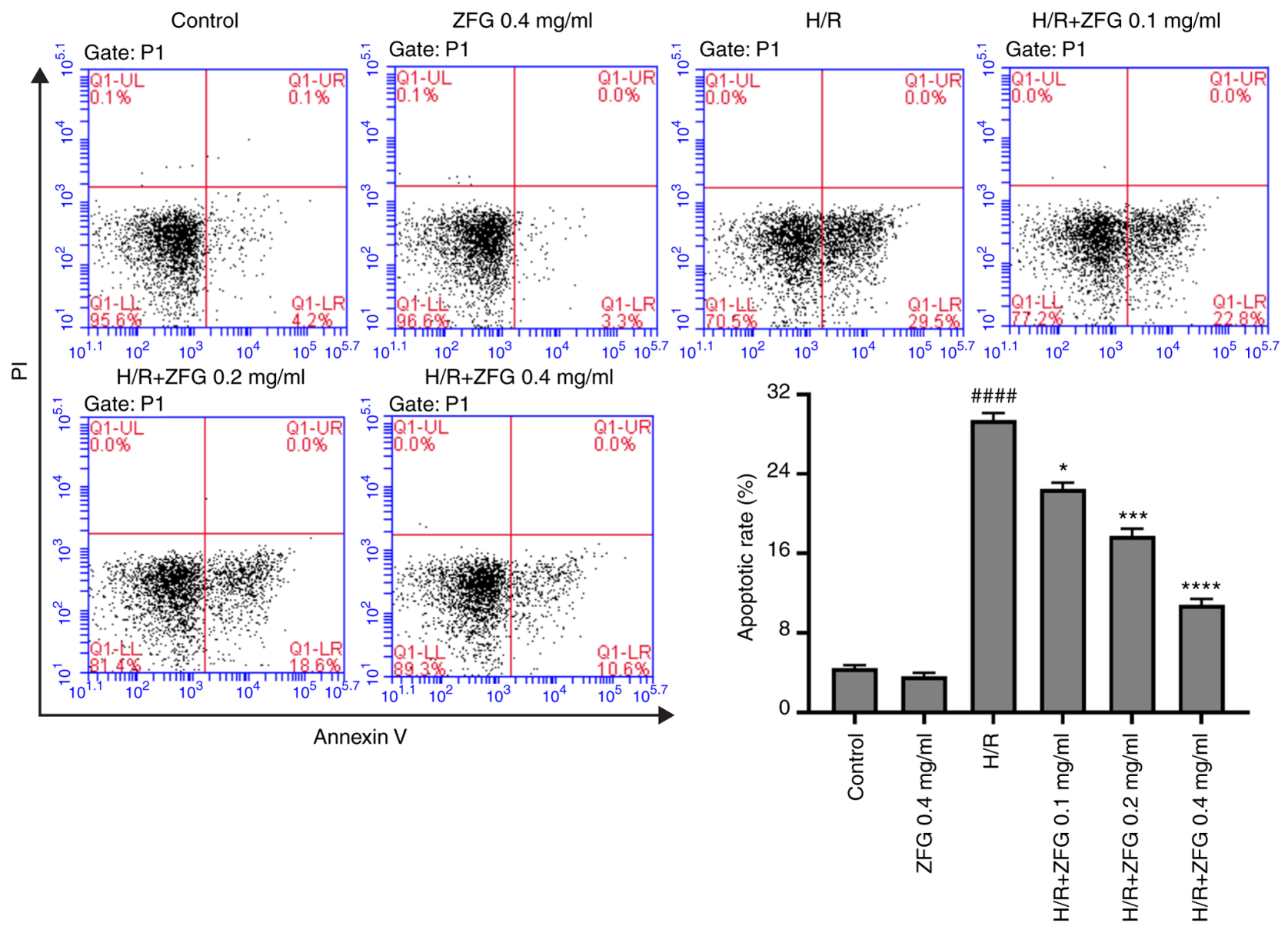

Figure 1.ZFG inhibits H/R-induced apoptosis in AC16 cells. H/R-induced AC16 cells were treated with ZFG (0.0, 0.1, 0.2 and $0.4 \mathrm{mg} / \mathrm{ml})$. After $24 \mathrm{~h}$, apoptotic cells were detected by flow cytometer. ${ }^{\# \# \# / P} \mathrm{P}<0.0001$ vs. Control; ${ }^{*} \mathrm{P}<0.05,{ }^{* * * *} \mathrm{P}<0.001$ and ${ }^{* * * * *} \mathrm{P}<0.0001$ vs. H/R. ZFG, Zengly Fumai Granule; H/R, hypoxia/ reoxygenation.

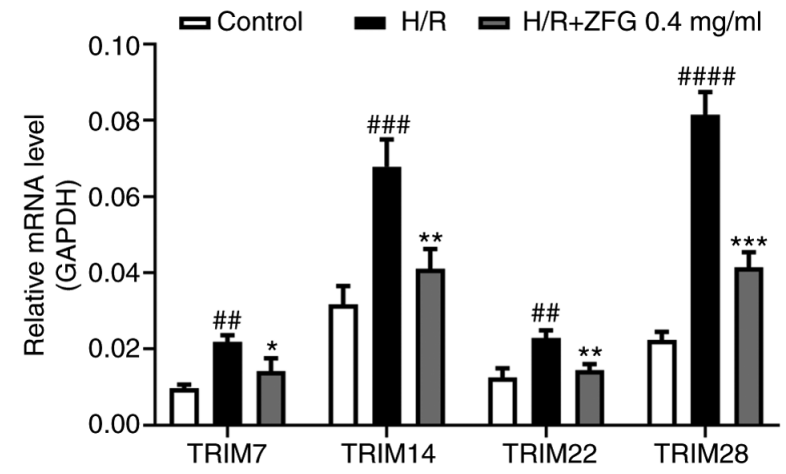

Figure 2. ZFG treatment inhibits H/R-induced upregulation of TRIM7, TRIM14, TRIM22 and TRIM28. H/R-induced AC16 cells were treated with $0.4 \mathrm{mg} / \mathrm{ml} \mathrm{ZFG}$. After $24 \mathrm{~h}$, the mRNA levels of TRIM7, TRIM14, TRIM22 and TRIM28 were detected by reverse transcription-quantitative PCR. ${ }^{\# \#} \mathrm{P}<0.01,{ }^{\# \# \#} \mathrm{P}<0.001$ and ${ }^{\# \# \#} \mathrm{P}<0.0001$ vs. Control; ${ }^{*} \mathrm{P}<0.05,{ }^{* *} \mathrm{P}<0.01$ and ${ }^{* * *} \mathrm{P}<0.001$ vs. H/R. ZFG, Zengly Fumai Granule; H/R, hypoxia/reoxygenation; TRIM, tripartite interaction motif.

H/R-induced upregulation of cleaved caspase-3 in AC16 cells (Fig. 4C). Thus, TRIM28 knockdown inhibited H/R-induced apoptosis in AC16 cells.

TRIM28 induces apoptosis in H/R-injured ACl6 cells by activating ROS generation. ROS production is a cause of myocardial apoptosis during MIR injury (23). Therefore, it was investigated whether TRIM28 promoted apoptosis by regulating ROS generation. The lentiviruses siTRIM28-1 and siTRIM28-2 were transduced into H/R-injured AC16 cells. $\mathrm{H} / \mathrm{R}$ treatment upregulated ROS levels in AC16 cells, while TRIM28 knockdown inhibited H/R-induced upregulation of ROS levels (Fig. 5A). In addition, lentivirus oeTRIM28 was used to overexpress TRIM28 in AC16 cells (Fig. S1A and B). TRIM28 overexpression increased the ROS levels and apoptotic rate in AC16 cells (Fig. 5B and C). However, the effects of oeTRIM28 on ROS and apoptosis were attenuated by ROS inhibitor NAC (Fig. 5B and C). Collectively, these data indicated that TRIM28 may promote apoptosis in H/R-injured AC16 cells via inducing ROS generation.

TRIM28 negatively regulates GPX1 stability via ubiquitination. GPX1 is an antioxidant enzyme that scavenges free radicals in cells and serves a protective role in MIR injury (24). In H/R-induced AC16 cells, the mRNA and protein levels of GPX1 were significantly downregulated (Fig. 6A). TRIM28 knockdown restored GPX1 protein levels but had no effect on GPX1 mRNA expression levels in H/R-induced AC16 cells (Fig. 6A). oeTRIM28 caused similar effects (Fig. 6B). Co-immunoprecipitation analysis revealed that TRIM28 interacted with GPX1 (Fig. 6C). Ubiquitination assays revealed 


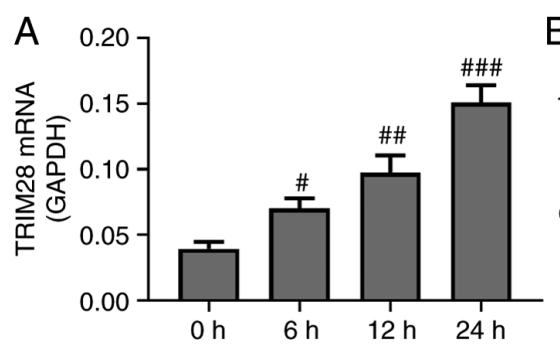

B
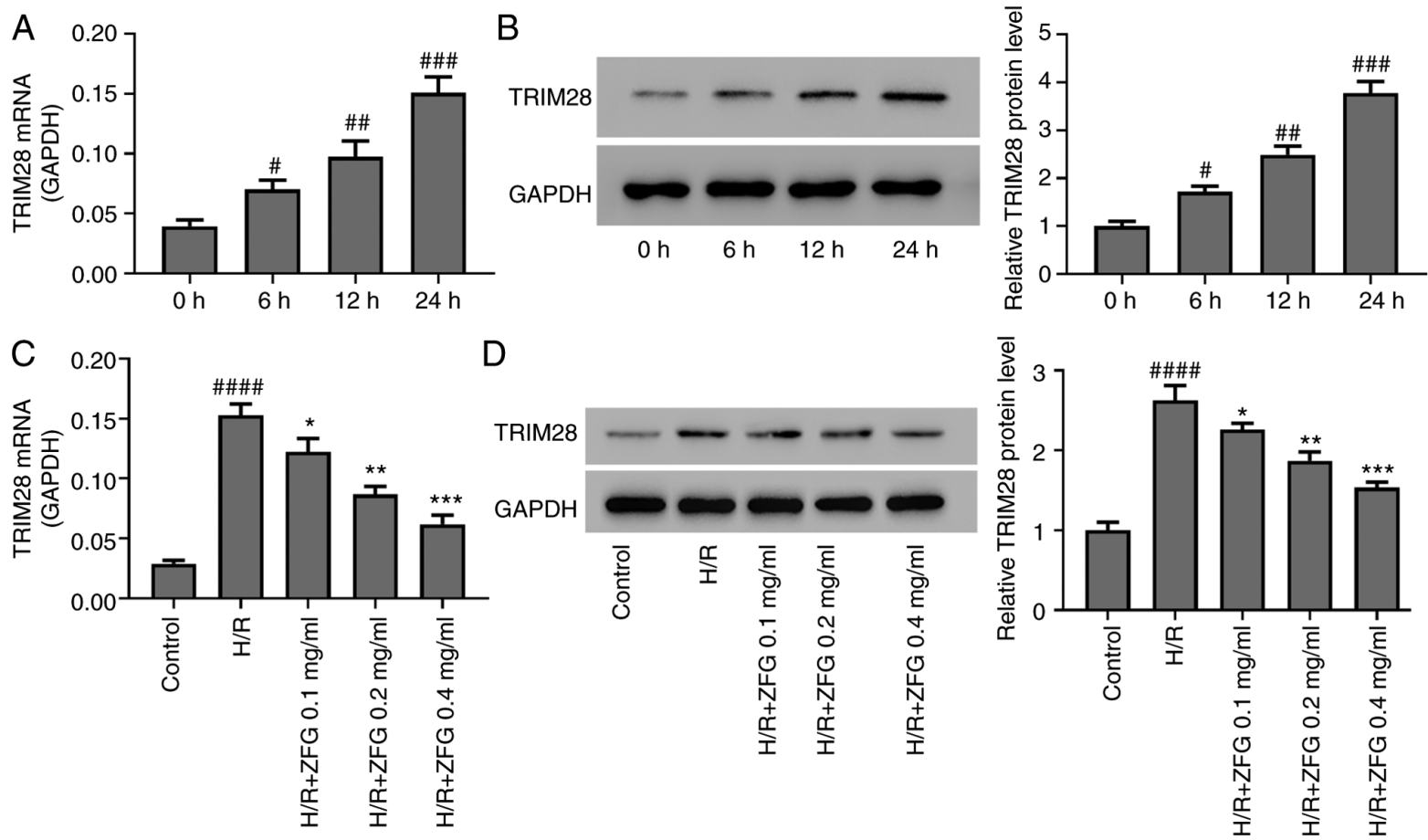

Figure 3. ZFG inhibits H/R-induced TRIM28 expression in AC16 cells. (A) mRNA and (B) protein levels of TRIM28 were measured by RT-qPCR and western blotting, respectively, at $0,6,12$ and $24 \mathrm{~h}$ after $\mathrm{H} / \mathrm{R}$ treatment. ${ }^{"} \mathrm{P}<0.05,{ }^{\# \#} \mathrm{P}<0.01$ and ${ }^{\# \# \#} \mathrm{P}<0.001$ vs. $0 \mathrm{~h}$. H/R-induced AC16 cells were treated with various concentrations of ZFG $(0.0,0.1,0.2$ and $0.4 \mathrm{mg} / \mathrm{ml})$. After $24 \mathrm{~h}$, the (C) mRNA and (D) protein levels of TRIM28 were measured by RT-qPCR and western blotting, respectively. ${ }^{\# \# \# /} \mathrm{P}<0.0001$ vs. Control; ${ }^{*} \mathrm{P}<0.05,{ }^{* * *} \mathrm{P}<0.01$ and ${ }^{* * * *} \mathrm{P}<0.0001$ vs. H/R. ZFG, Zengly Fumai Granule; H/R, hypoxia/reoxygenation; TRIM, tripartite interaction motif; RT-q, reverse transcription-quantitative.

A
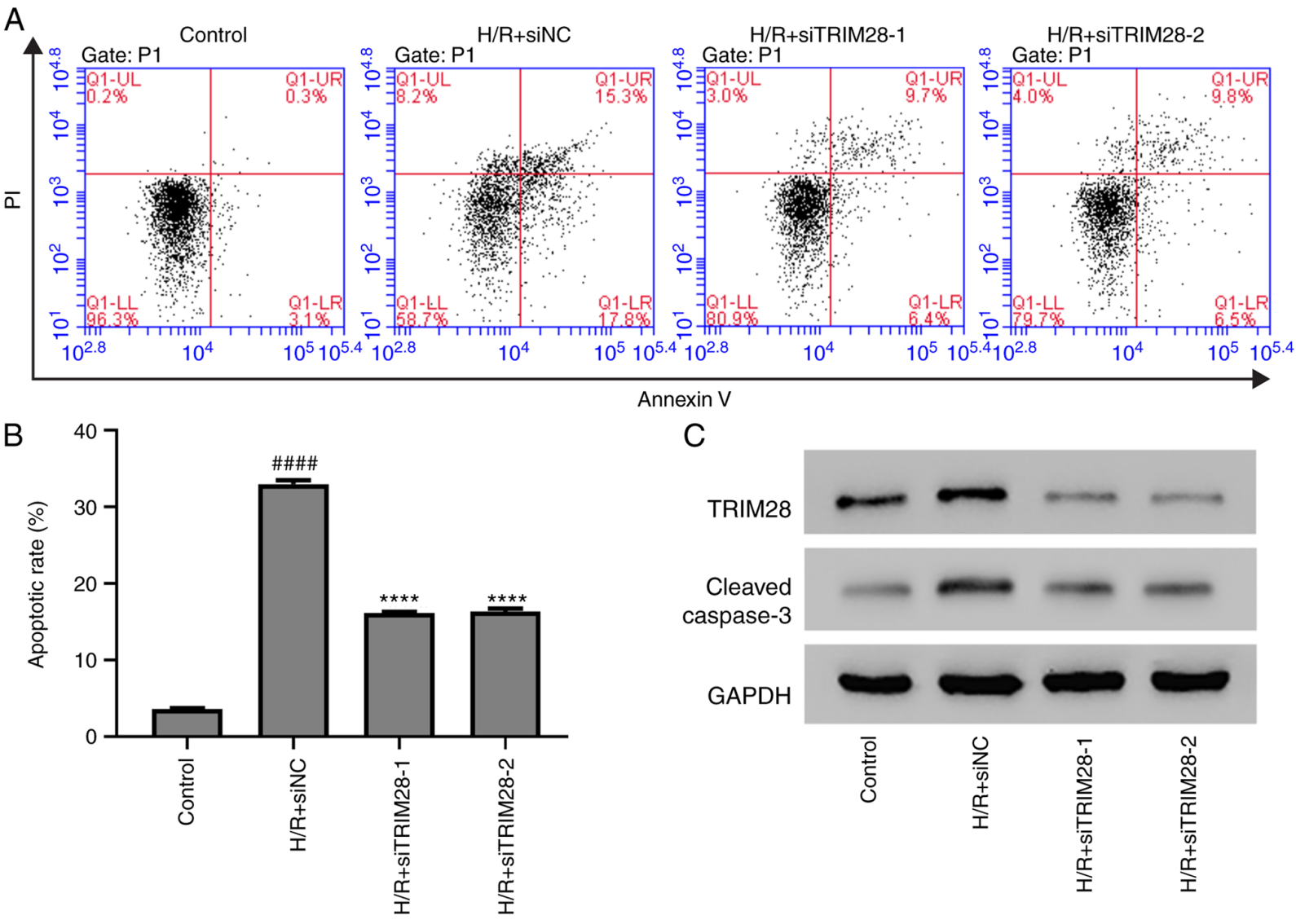

Annexin V

C

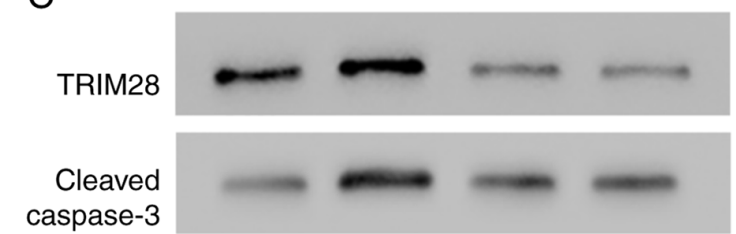

GAPDH

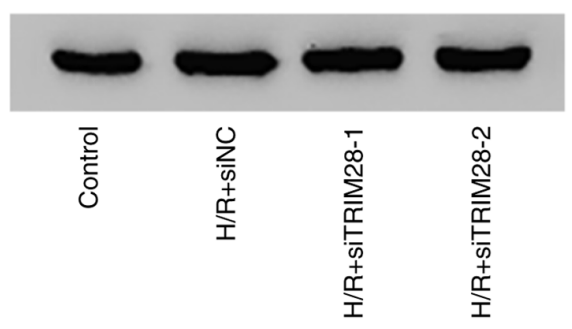

Figure 4. Knockdown of TRIM28 inhibits H/R-induced apoptosis in AC16 cells. H/R-induced AC16 cells were transduced with lentivirus siTRIM28 or siNC, and cultured for 24 h. (A) Apoptotic cells were detected by flow cytometer. (B) The apoptotic rate. (C) Protein levels of TRIM28 and cleaved caspase-3 were measured by western blotting. ${ }^{\# \# \# " ~} \mathrm{P}<0.0001$ vs. Control; ${ }^{* * * * *} \mathrm{P}<0.0001 \mathrm{vs.} \mathrm{H/R}+$ siNC. TRIM, tripartite interaction motif; H/R, hypoxia/reoxygenation; si, small interfering RNA; $\mathrm{NC}$, negative control. 
A
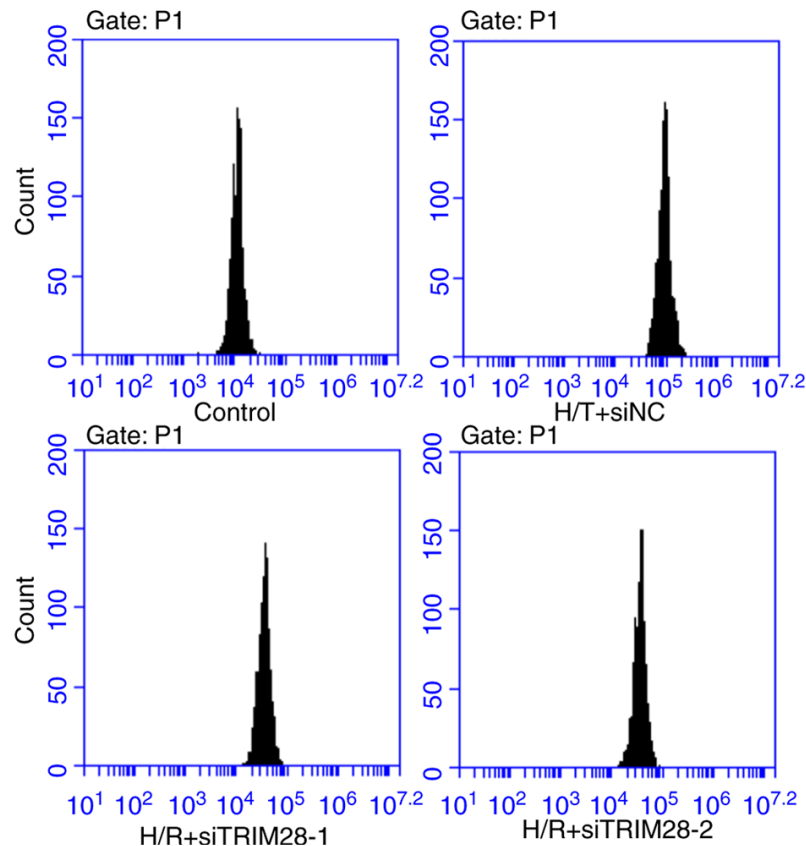

H/R+siTRIM28-2

B
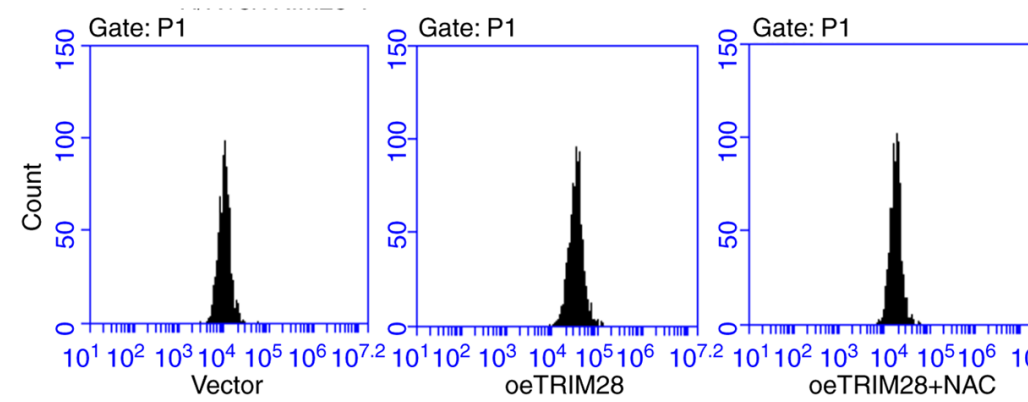
oeTRIM28
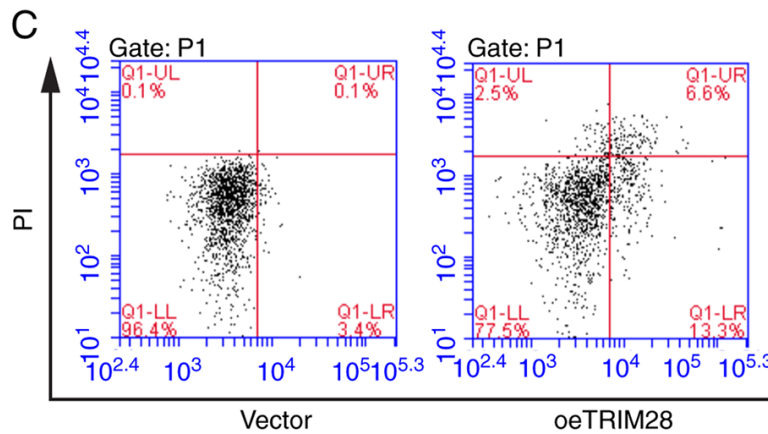

oeTRIM28

$10^{1} 10^{2} 10^{3}$ OETRIM28+NAC
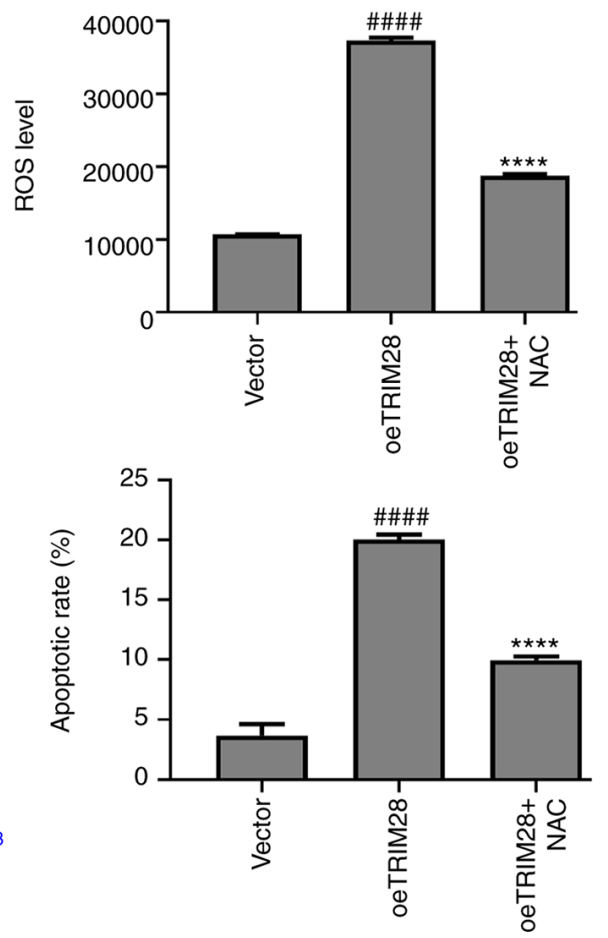

Figure 5. TRIM28 enhances apoptosis in AC16 cells by activating ROS generation. (A) H/R-induced AC16 cells were transduced with lentivirus siTRIM28

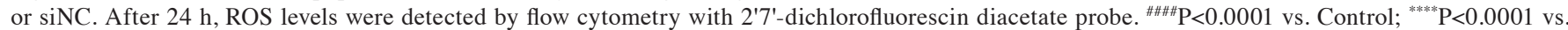
$\mathrm{H} / \mathrm{R}+$ siNC. AC16 cells were transduced with oeTRIM28 and cultured in the presence or absence of $1 \mathrm{mmol} / 1$ ROS inhibitor NAC for $24 \mathrm{~h}$. (B) ROS levels and (C) apoptotic cells were measured by flow cytometry. ${ }^{\# \# \# ~} \mathrm{P}<0.0001$ vs. Vector; ${ }^{* * * * *} \mathrm{P}<0.0001$ vs. oeTRIM28. TRIM, tripartite interaction motif; ROS, reactive oxygen species; H/R, hypoxia/reoxygenation; si, small interfering RNA; NC, negative control; NAC, N-acetyl-L-cysteine; oe, overexpression.

that oeTRIM28 promoted the ubiquitination of GPX1 in AC16 cells (Fig. 6D). Collectively, these data indicated that TRIM28 negatively regulated GPX1 via ubiquitination.

\section{Discussion}

ZFG is a clinical prescription for the treatment of sick sinus syndrome (12). Previous studies have revealed a close association between sick sinus syndrome and MIR injury $(14,25)$. Therefore, it was speculated that ZFG may exert a protective effect on MIR injury. The present study revealed that ZFG significantly inhibited H/R-induced cardiomyocyte apoptosis, supporting this hypothesis.

A recent study demonstrated that Tongxinluo capsule, a Chinese herbal compound, attenuates MIR injury by downregulating the ubiquitin-proteasome system (26). Previous studies have also revealed that the ubiquitin-proteasome system serves an important regulatory role in MIR injury $(17,18)$. Hence, it was hypothesized that ZFG may also exert a protective effect by regulating the ubiquitin-proteasome system. TRIM proteins, 

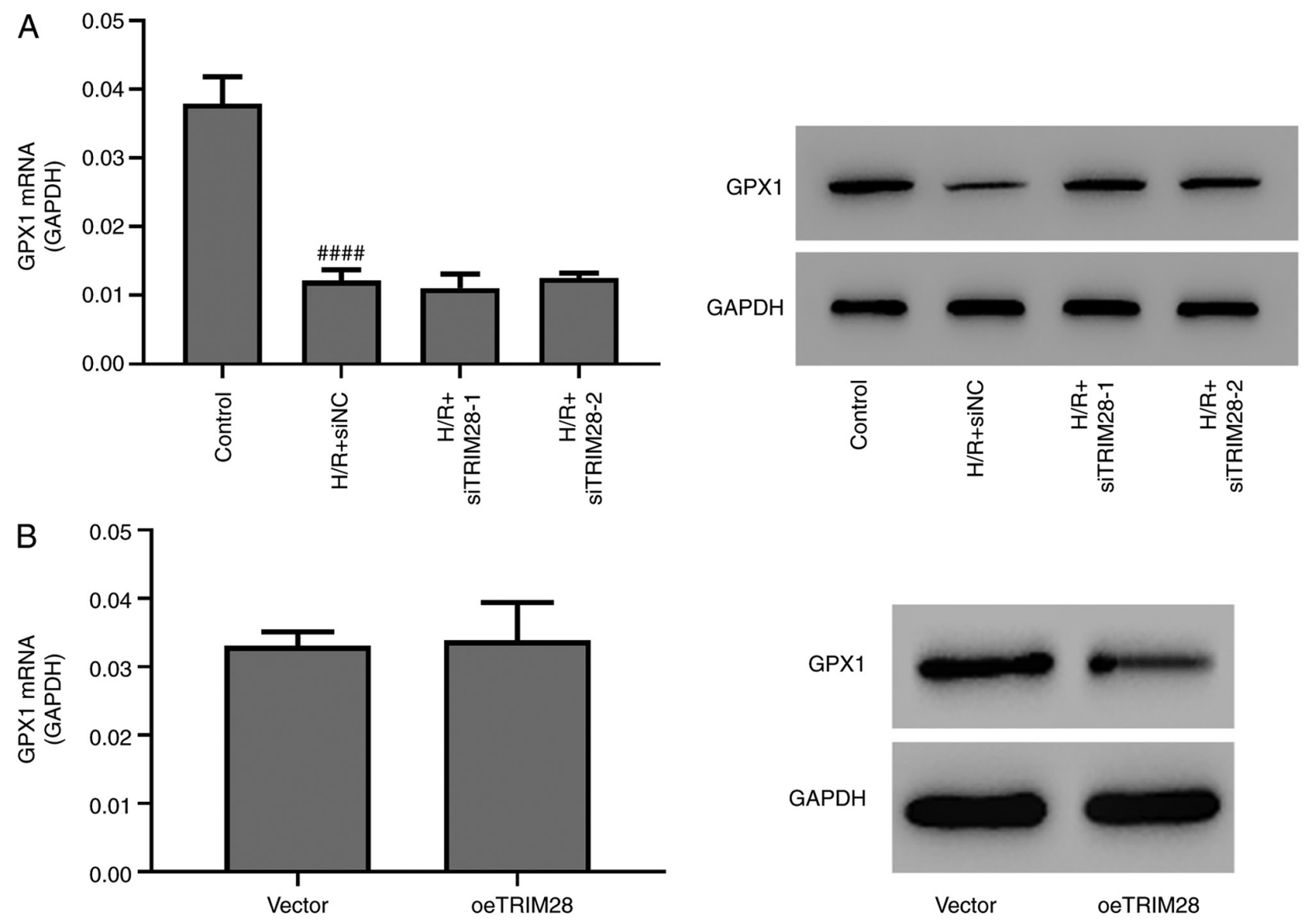

GPX1

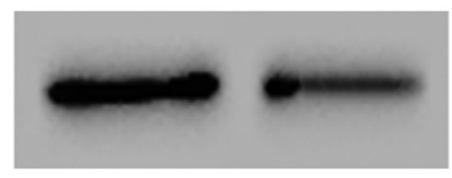

GAPDH

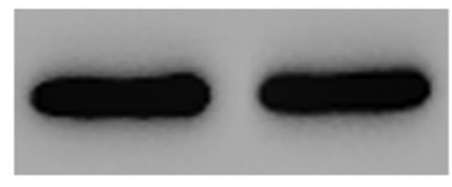

Vector

oeTRIM28

C

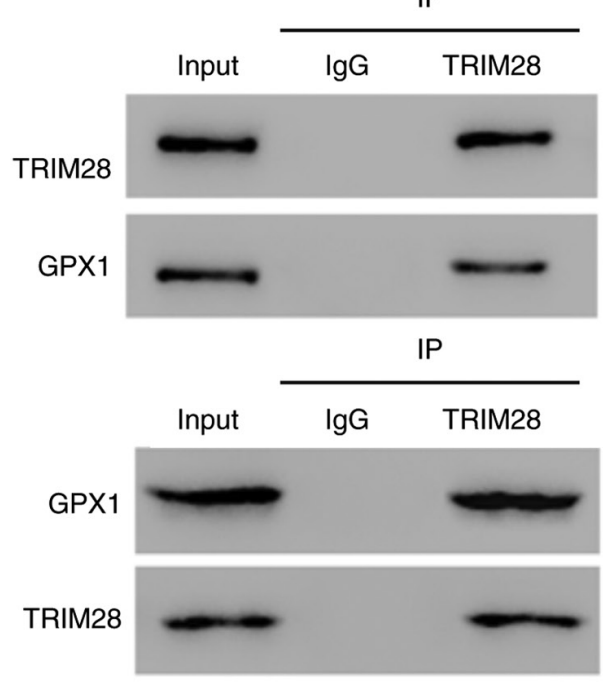

D

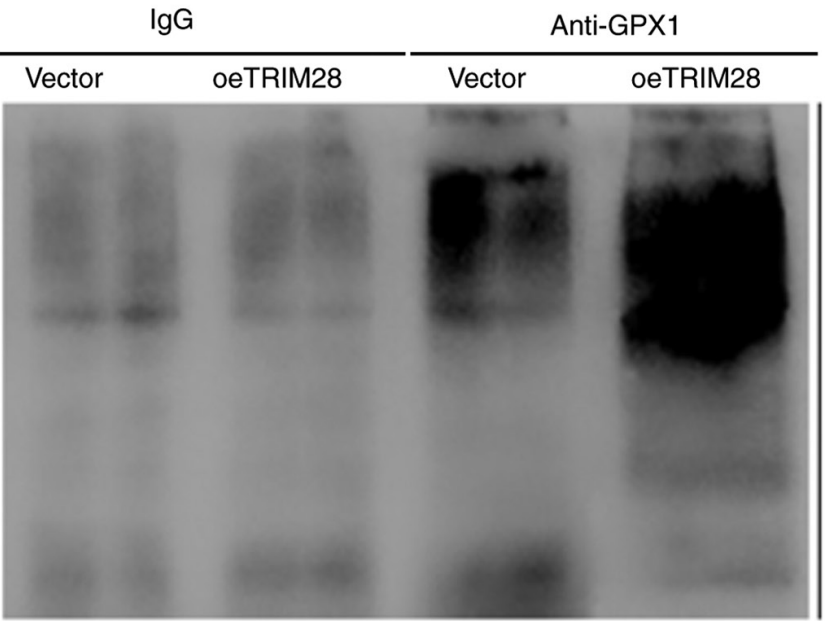

Figure 6. TRIM28 negatively regulates GPX1 stability via ubiquitination. (A) H/R-induced AC16 cells were transduced with lentivirus siTRIM28 or siNC and (B) AC16 cells were transduced with lentivirus oeTRIM28. After $24 \mathrm{~h}$, the mRNA and protein levels of GPX1 were measured by reverse transcriptionquantitative PCR and western blotting, respectively. (C) Interaction of TRIM28 and GPX1 was detected by co-immunoprecipitation. (D) GPX1 antibody was used to pull down immunocomplex. Ubiquitin antibody was used for subsequent western blotting. ${ }^{\# \# \# P<0.0001 ~ v s . ~ C o n t r o l . ~ T R I M, ~ t r i p a r t i t e ~ i n t e r a c t i o n ~ m o t i f ; ~}$ GPX, glutathione peroxidase; H/R, hypoxia/reoxygenation; si, small interfering RNA; NC, negative control; oe, overexpression.

which mediate the ubiquitin-dependent protein degradation pathway, are the primary members of ubiquitin-proteasome system (27). A number of TRIM proteins are expressed abnormally in the heart in patients with myocardial infarction, including TRIM7, TRIM14, TRIM22 and TRIM28 (19). The present study assessed the expression levels of these four proteins in cardiomyocytes following $\mathrm{H} / \mathrm{R}$ and ZFG treatment.
These proteins were significantly upregulated in H/R-injured cardiomyocytes, whereas their expression was inhibited by subsequent administration of ZFG. Of these four proteins, TRIM28 induced the greatest change in expression levels and was therefore selected for subsequent analysis. Silencing TRIM28 attenuated H/R-induced apoptosis. Therefore, ZFG may protect cardiomyocytes against H/R-induced apoptosis 
partly by regulating TRIM28. Further research is necessary to confirm the involvement of TRIM7, TRIM14 and TRIM22 in the protective role of ZFG in MIR injury.

TRIM28, also called TIF1 $\beta$ or KAP1, is involved in multiple cellular processes, such as proliferation, migration and apoptosis $(28,29)$. It has also been revealed to regulate apoptosis in a cell type-dependent manner. For example, TRIM28 serves an anti-apoptotic function in immature erythroid, lymphoma and lung cancer cells (30-32) but exerts a pro-apoptotic effect on bovine fibroblasts and embryonic kidney cells $(33,34)$. The present study revealed that oeTRIM28 promoted apoptosis in AC16 cells, suggesting a pro-apoptotic role of TRIM28 in cardiomyocytes. Previous research has primarily focused on the role of TRIM28 in cancer $(35,36)$. To the best of our knowledge, the role of TRIM28 in MIR injury has not been studied. The present study, reported the involvement of TRIM28 in an in vitro MIR model. TRIM28 was also revealed to be upregulated and induce apoptosis in H/R-injured cardiomyocytes.

During MIR injury, excessive ROS are produced, triggering a series of pathological processes, including cardiomyocyte apoptosis and the inflammatory response (23). Therefore, ROS have been recognized as critical regulators in MIR injury. As anticipated, ROS levels in H/R-injured cardiomyocytes were significantly upregulated in the present study. However, TRIM28 knockdown attenuated H/R-induced accumulation of ROS in cardiomyocytes. Previous studies have revealed that numerous TRIM proteins, such as TRIM10 and TRIM69, promote cell apoptosis via enhancing ROS generation $(37,38)$. Consistent with these results, in the present study, TRIM28 promoted cardiomyocyte apoptosis by regulating ROS production. GPX1 is an antioxidant enzyme that can directly scavenge ROS in cells (39), and thereby negatively regulate ROS. The present study revealed that GPX1 was significantly decreased in H/R-injured cardiomyocytes, which is consistent with previous studies $(40,41)$. TRIM 28 knockdown restored GPX1 protein levels but had no effect on GPX1 mRNA levels in $\mathrm{H} / \mathrm{R}$-injured cardiomyocytes. A previous study reported that TRIM33 was involved in MIR injury by ubiquitinating GPX1 (24). In the present study, TRIM28 negatively regulated GPX1 via ubiquitination. Collectively, the present results indicated that TRIM28 may serve a pro-apoptotic role in $\mathrm{H} / \mathrm{R}$-injured cardiomyocytes by regulating the GPX1/ROS pathway.

In summary, the present study demonstrated that ZFG attenuated H/R-induced cardiomyocyte apoptosis via the inhibition of TRIM28 expression levels. TRIM28 served a pro-apoptotic role in cardiomyocytes by enhancing ROS generation via GPX1 ubiquitination. These results suggested that ZFG and TRIM28 are potential drug and therapeutic targets for MIR injury treatment, respectively. Further in vivo research is necessary to confirm the present findings.

\section{Acknowledgements}

Not applicable.

\section{Funding}

The present study was supported by the Science and Technology Major Projects of Jilin Province: Study on the treatment of sick sinus syndrome by Zenglv Fumai Granule (grant no. 20160204024YY).

\section{Availability of data and materials}

The datasets used and/or analyzed during the current study are available from the corresponding author on reasonable request.

\section{Authors' contributions}

XHZ and SRL designed the study. HYZ, XYL and FQ performed the experiments. XHZ and SRL confirmed the authenticity of all the raw data. YW and LD analyzed and interpreted the data. All authors wrote the manuscript and read and approved the final manuscript.

\section{Ethics approval and consent to participate}

Not applicable.

\section{Patient consent for publication}

Not applicable.

\section{Competing interests}

The authors declare that they have no competing interests.

\section{References}

1. WHO: The Top 10 Causes of Death. World Health Organization, Geneva, 2018. https://www.who.int/news-room/fact-sheets/ detail/the-top-10-causes-of-death.

2. Yellon DM and Hausenloy DJ: Myocardial reperfusion injury. N Engl J Med 357: 1121-1135, 2007.

3. Buja LM: Myocardial ischemia and reperfusion injury. Cardiovasc Pathol 14: 170-175, 2005.

4. Hausenloy DJ and Yellon DM: Myocardial ischemia-reperfusion injury: A neglected therapeutic target. J Clin Invest 123: 92-100, 2013.

5. Ye G, Fu Q, Jiang L and Li Z: Vascular smooth muscle cells activate PI3K/Akt pathway to attenuate myocardial ischemia/ reperfusion-induced apoptosis and autophagy by secreting bFGF. Biomed Pharmacother 107: 1779-1785, 2018.

6. Zhao D, Feng P, Sun Y, Qin Z, Zhang Z, Tan Y, Gao E, Lau WB, Ma X, Yang J, et al: Cardiac-derived CTRP9 protects against myocardial ischemia/reperfusion injury via calreticulindependent inhibition of apoptosis. Cell Death Dis 9: 723, 2018.

7. Zhou H, Zhu P, Guo J, Hu N, Wang S, Li D, Hu S, Ren J, Cao F and Chen Y: Ripk3 induces mitochondrial apoptosis via inhibition of FUNDC1 mitophagy in cardiac IR injury. Redox Biol 13: 498-507, 2017.

8. Liu Q, Li J, Wang J, Li J, Janicki JS and Fan D: Effects and mechanisms of chinese herbal medicine in ameliorating myocardial ischemia-reperfusion injury. Evid Based Complement Alternat Med 2013: 925625, 2013.

9. Vincent A, Covinhes A, Barrère C, Gallot L, Thoumala S, Piot C, Heurteaux C, Lazdunski M, Nargeot J and Barrère-Lemaire S: Acute and long-term cardioprotective effects of the traditional Chinese medicine MLC901 against myocardial ischemiareperfusion injury in mice. Sci Rep 7: 14701, 2017.

10. Zhao J, Huang X, Tang W, Ren P, Xing Z, Tian X, Zhu Z and Wang Y: Effect of oriental herbal prescription Guan-Xin-Er-Hao on coronary flow in healthy volunteers and antiapoptosis on myocardial ischemia-reperfusion in rat models. Phytother Res 21: 926-931, 2007.

11. Zhao Y, Xu L, Qiao Z, Gao L, Ding S, Ying X, Su Y, Lin N, He B and Pu J: YiXin-Shu, a ShengMai-San-based traditional Chinese medicine formula, attenuates myocardial ischemia/reperfusion injury by suppressing mitochondrial mediated apoptosis and upregulating liver-X-receptor $\alpha$. Sci Rep 6: 23025, 2016. 
12. Zhang X, Yu D, Qian F and Liu S: Zenglv Fumai Granule on 120 cases of sick sinus syndrome. J Tradit Chin Med 26: 1926-1928, 2017.

13. Jou CJ, Arrington CB, Barnett S, Shen J, Cho S, Sheng X, McCullagh PC, Bowles NE, Pribble CM, Saarel EV, et al: A functional assay for sick sinus syndrome genetic variants. Cell Physiol Biochem 42: 2021-2029, 2017.

14. Zhang W, Zhu B, Ren J, Lu F, Qi Y, Weng W and Gao R: Two methods for modeling of sick sinus syndrome in rats: Ischemia reperfusion and sodium hydroxide induced injury. Biomed Pharmacother 111: 778-784, 2019.

15. Watanabe M and Hatakeyama S: TRIM proteins and diseases. J Biochem 161: 135-144, 2017

16. Yamada Y, Takayama KI, Fujimura T, Ashikari D, Obinata D, Takahashi S, Ikeda K, Kakutani S, Urano T, Fukuhara H, et al: A novel prognostic factor TRIM44 promotes cell proliferation and migration, and inhibits apoptosis in testicular germ cell tumor. Cancer Sci 108: 32-41, 2017.

17. Zeng $G$, Lian $C$, Yang $P$, Zheng $M$, Ren $H$ and Wang $H$ : E3-ubiquitin ligase TRIM6 aggravates myocardial ischemia/ reperfusion injury via promoting STAT1-dependent cardiomyocyte apoptosis. Aging (Albany NY) 11: 3536-3550, 2019.

18. Lv ZQ, Yang CY and Xing QS: TRIM59 attenuates inflammation and apoptosis caused by myocardial ischemia reperfusion injury by activating the PI3K/Akt signaling pathway. Eur Rev Med Pharmacol Sci 24: 5192-5192, 2020.

19. Borlepawar A, Frey N and Rangrez AY: A systematic view on E3 ligase Ring TRIMmers with a focus on cardiac function and disease. Trends Cardiovasc Med 29: 1-8, 2019

20. Zhang D, Zhang X and Wang Z: Optimization of extraction techenology of Zengmaifulv Granules by orthogonal test. Shandong Chem Ind 45: 28-30, 2016.

21. Benoist L, Chadet S, Genet T, Lefort C, Heraud A, Danila MD, Muntean DM, Baron C, Angoulvant D, Babuty D, et al: Stimulation of P2Y11 receptor protects human cardiomyocytes against Hypoxia/Reoxygenation injury and involves $\mathrm{PKC} \varepsilon$ signaling pathway. Sci Rep 9: 11613, 2019.

22. Livak KJ and Schmittgen TD: Analysis of relative gene expression data using real-time quantitative PCR and the $2(-\Delta \Delta$ C(T)) Method. Methods 25: 402-408, 2001.

23. Zhao ZQ: Oxidative stress-elicited myocardial apoptosis during reperfusion. Curr Opin Pharmacol 4: 159-165, 2004.

24. Jian Z, Liang B, Pan X, Xu G, Guo SS, Li T, Zhou T, Xiao YB and Li AL: CUEDC2 modulates cardiomyocyte oxidative capacity by regulating GPX1 stability. EMBO Mol Med 8: 813-829, 2016

25. Liu RX, Tan S, Liu M, Peng J, Wang YL and Liu ZM: Effects of Chinese herbal medicine serum on the apoptosis of sinoatrial node cells induced by simulated ischemia-reperfusion. J Tradit Chin Med 31: 224-227, 2011

26. Yang HX, Wang P, Wang NN, Li SD and Yang MH: Tongxinluo ameliorates myocardial ischemia-reperfusion injury mainly via activating parkin-mediated mitophagy and downregulating ubiquitin-proteasome system. Chin J Integr Med: June, 2019 DOI: $10.1007 / \mathrm{s} 11655-019-3166-8$.

27. Hatakeyama S: TRIM family proteins: Roles in autophagy, immunity, and carcinogenesis. Trends Biochem Sci 42: 297-311, 2017.
28. Liu H, Chen H, Deng X, Peng Y, Zeng Q, Song Z, He W, Zhang L, Xiao T, Gao G, et al: Knockdown of TRIM28 inhibits PDGF-BBinduced vascular smooth muscle cell proliferation and migration. Chem Biol Interact 311: 108772, 2019.

29. Peng Y, Zhang M, Jiang Z and Jiang Y: TRIM28 activates autophagy and promotes cell proliferation in glioblastoma. OncoTargets Ther 12: 397-404, 2019.

30. Zhang P-P, Ding D-Z, Shi B, Zhang SQ, Gu LL, Wang YC and Cheng C: Expression of TRIM28 correlates with proliferation and Bortezomib-induced apoptosis in B-cell non-Hodgkin lymphoma. Leuk Lymphoma 59: 2639-2649, 2018.

31. Liu L, Zhang L, Wang J, Zhao X, Xu Q, Lu Y, Zuo Y, Chen L, Du J, Lian Y, et al: Downregulation of TRIM28 inhibits growth and increases apoptosis of nude mice with non-small cell lung cancer xenografts. Mol Med Rep 17: 835-842, 2018.

32. Hosoya T, Clifford M, Losson R, Tanabe O and Engel JD TRIM28 is essential for erythroblast differentiation in the mouse. Blood 122: 3798-3807, 2013.

33. Ma P, Man X, Yang S, Dong X, Su L, Luan W and Ma X: Effect of TRIM28 on proliferation, apoptosis and histone H3K9 trimethylation in bovine fibroblasts. Indian J Animal Res 53: 724-730, 2019.

34. Lionnard L, Duc P, Brennan MS, Kueh AJ, Pal M, Guardia F, Mojsa B, Damiano MA, Mora S, Lassot I, et al: TRIM17 and TRIM28 antagonistically regulate the ubiquitination and antiapoptotic activity of BCL2A1. Cell Death Differ 26: 902-917, 2019.

35. Wei C, Cheng J, Zhou B, Zhu L, Khan MA, He T, Zhou S, He J, $\mathrm{Lu} \mathrm{X}$, Chen H, et al: Tripartite motif containing 28 (TRIM28) promotes breast cancer metastasis by stabilizing TWIST1 protein. Sci Rep 6: 29822, 2016.

36. 36. Fong KW, Zhao JC, Song B, Zheng B and Yu J: TRIM 28 protects TRIM24 from SPOP-mediated degradation and promotes prostate cancer progression. Nat Commun 9: 5007, 2018.

37. Huang Q, Zhu X and Xu M: Silencing of TRIM10 alleviates apoptosis in cellular model of Parkinson's disease. Biochem Biophys Res Commun 518: 451-458, 2019.

38. Rong X, Rao J, Li D, Jing Q, Lu Y and Ji Y: TRIM69 inhibits cataractogenesis by negatively regulating p53. Redox Biol 22: 101157, 2019.

39. Sies $\mathrm{H}$ : Role of metabolic $\mathrm{H} 2 \mathrm{O} 2$ generation: Redox signaling and oxidative stress. J Biol Chem 289: 8735-8741, 2014.

40. Seara FAC, Maciel L, Barbosa RAQ, Rodrigues NC, Silveira ALB, Marassi MP, Carvalho AB, Nascimento JHM and Olivares EL: Cardiac ischemia/reperfusion injury is inversely affected by thyroid hormones excess or deficiency in male Wistar rats. PLoS One 13: e0190355, 2018.

41. Jian Z, Liang B, Pan X, Xu G, Guo SS, Li T, Zhou T, Xiao YB and Li AL: CUEDC2 modulates cardiomyocyte oxidative capacity by regulating GPX1 stability. EMBO Mol Med 8: 813-829, 2016.

(i) $\ominus$ This work is licensed under a Creative Commons Attribution-NonCommercial-NoDerivatives 4.0 International (CC BY-NC-ND 4.0) License. 